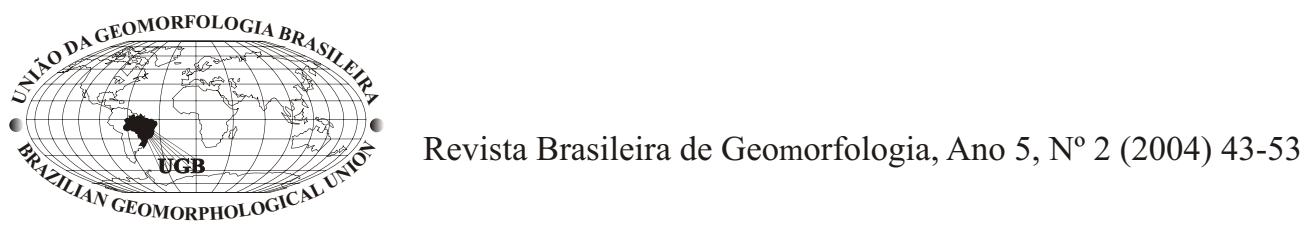

\title{
Calculo Preliminar de la Tasa de Meteorización del Batolito Antioqueño, Cordillera Central, Colombia
}

\author{
Cálculo Preliminar da Taxa de Intemperismo do Batólito Antioqueño, Cordilheira \\ Central, Colômbia
}

\author{
Carolina Garcia, Michel Hermelin
}

Universidad EAFIT, Grupo de Geología Ambiental e Ingeniería Sísmica. Tel. +5742619331 cgarci10@eafit.edu.co, hermelin@eafit.edu.co

\begin{abstract}
Resumen
Este trabajo es una estimación preliminar del tiempo necesario para generar la espesa capa de saprolito que caracteriza el perfil de meteorización actual de un batolito cuarzodiorítico cretácico que aflora en altiplanos de la Cordillera Central de Colombia, entre $5^{\circ} 50^{\prime}$ y $7^{\circ} 00^{\prime}$ de latitud Norte y $74^{\circ} 50^{\prime}$ y $75^{\circ} 35^{\prime}$ de longitud Oeste y a alturas entre 750 y $2700 \mathrm{~m}$. Se utilizan datos obtenidos previamente mediante el análisis de la composición química de las aguas superficiales de cuatro cuencas con áreas diferentes, ubicadas sobre el Batolito Antioqueño, al este de Medellín. Los cálculos se hicieron con el método basado en la pérdida de componentes en solución utilizando diferentes valores de solutos. Al utilizar la pérdida de sílice se obtiene un tiempo de meteorización de unos $6 \times 10^{6}$ años, comparable con el obtenido mediante la datación de las cenizas volcánicas mas antiguas que recubren la topografía actual y que indican la fecha mínima de configuración de la superficie de erosión.A pesar de resultar de una serie de suposiciones simplificadoras, este resultado es interesante y justifica la realización de determinaciones mas detalladas. Confirma la larga preservación de superficies de erosión antiguas conformadas sobre un saprolito que desde hace varios millones de años no ha sido sometido a una erosión profunda, fenómeno poco común en una zona tectónicamente activa como la estudiada.
\end{abstract}

Palabras claves: meteorización química, Batolito Antioqueño, Colombia

\begin{abstract}
This is a preliminary estimation of the time necessary to generate the thick layer of saprolite that characterizes the weathering profile derived from a cretaceous quartzodiorite batholith of the Colombian Central Cordillera, $5^{\circ} 50$ a to $7^{\circ} 00 \mathrm{~N}$ latitude and $74^{\circ} 50$ a $75^{\circ} 35 \mathrm{~W}$ longitude at altitudes between 750 and $2700 \mathrm{~m}$. We used data from analyses of the chemical compositions of surface waters from four river basins with different areas draining the batholith. Calculations were based on the loss of several components in solution. The loss of silica permits to calculate a weathering time of about $6 \times 106$ years, comparable with the one obtained by dating the olderst volcanic ashes that cover the present topography.Despite of several simplifying assumptions, this result is interesting and justifies future more detailed determinations. It confirms the long preservation of old erosion surfaces derived from saprolite that for several million years have not been exposed to deep erosion, a phenomenon which is not common in a tectonically active zone as the studied one.
\end{abstract}

Key words: chemical weathering, Antioquian Batholith, Colombia 


\section{Introducción}

La meteorización química es el conjunto de procesos y de reacciones químicas que transforma la roca en materiales más estables ante los agentes meteóricos como el agua y el aire en la superficie terrestre (Colman, 1986).

Este tipo de meteorización es mas intenso en los trópicos húmedos donde las altas temperaturas y la abundante precipitación aceleran las reacciones y aumentan la remoción de cationes de los minerales. La tasa de meteorización depende de la composición de la roca, así como del clima y de la actividad orgánica; puede ser interpretada como la tasa de penetración de la meteorización en la masa de roca (tasa de producción de saprolito) o como la tasa de transformación de minerales a otros que estén en mayor equilibrio con el ambiente de aguas infiltradas (tasa de alteración) (Thomas, 1994b). En este estudio se parte de la transformación de los minerales primarios (principalmente silicatos) de una roca granodiorítica (Batolito Antioqueño) cuya meteorización química se evalúa en cada cuenca por la cantidad de sílice y otros elementos disueltos en las aguas superficiales en exceso de la cantidad introducida por la lluvia. (Cleaves, 1970).

El estudio de la dinámica de la meteorización permite habitualmente entender la evolución del paisaje ya que el espesor del saprolito depende de la susceptibilidad del paisaje a la erosión física. En el caso estudiado el paisaje ha permanecido estable a pesar de la profunda capa meteorizada.

\section{Localización}

Los datos utilizados en este trabajo proceden de cuatro cuencas con diferentes áreas, geográficamente separadas, ubicadas en el Municipio de San Carlos $\left(75^{\circ} \mathrm{W} 6.2^{\circ} \mathrm{N}\right)$, al sureste de Medellín (Fig. 1), sobre rocas del Batolito Antioqueño.

La temperatura promedio va de 18 a $26^{\circ} \mathrm{C}$ y no varía durante el año pero sí entre el día y la noche. La precipitación promedio anual es de 3500 a $4700 \mathrm{~mm}$.

En la Tabla 1 se dan los datos más representativos para el estudio de las cuatro cuencas.

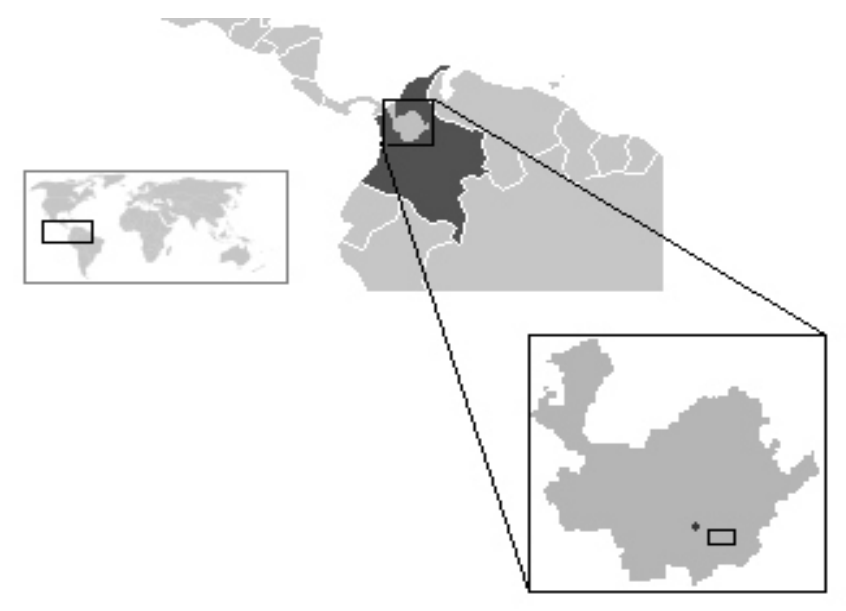

Figura 1. Localización del área de estudio

Tabla 1. Hidrología, pluviometría, aspectos topográficos y áreas de las diferentes cuencas estudiadas

\begin{tabular}{|c|c|c|c|c|}
\hline & Río Calderas & Río Tafetanes & $\begin{array}{c}\text { Quebrada } \\
\text { Jaguas }\end{array}$ & $\begin{array}{c}\text { Quebrada } \\
\text { Juanes }\end{array}$ \\
\hline Area Cuenca, ha & 4086 & 1118.5 & 778.98 & 16 \\
\hline Cobertura Vegetal* & 49 & 22 & 9 & 17 \\
\hline Elevación Max., msnm & 2250 & 2200 & 1400 & 800 \\
\hline Elevación Min., msnm & 1535 & 1929 & 1100 & 750 \\
\hline Elev. Prom., msnm & 1900 & 2050 & 1150 & 750 \\
\hline Pluviosidad, mm/año** & 3986.6 & 4656 & 3565.6 & 4605.5 \\
\hline Caudal, l/s & 3900 & 1060 & 640 & 6 \\
\hline Relación Area/Caudal & 1.048 & 1.055 & 1.217 & 2.667 \\
\hline
\end{tabular}

* Porcentaje del área cubierto por bosque, medido con planímetro sobre mapa del IGAC, 1979

** Datos promedio 1984-1987 (Instituto Colombiano de Hidrología, Meteorología y Adecuación de Tierras -HIMAT) 


\section{Geología}

El Batolito Antioqueño es un cuerpo intrusivo de composición granodiorítica de edad cretácica que aflora en un área de $7221 \mathrm{~km}^{2}$ localizada en la Cordillera Central de Colombia, a alturas entre 750 y $2700 \mathrm{~m}$ sobre el nivel del mar.
Es interpretado como una gran intrusión de manto subhorizontal de espesor relativamente pequeño con relación al área expuesta (Feininger \& Botero, 1982). Uno de los aspectos mas relevantes del batolito es su homogeneidad petrográfica, con el $97 \%$ de su composición correspondiente a granodiorita o cuarzodiorita (tabla 2).

Tabla 2. Composición mineralógica promedio del Batolito Antioqueño (Feininger \& Botero, 1982)

\begin{tabular}{|l|c|}
\hline MINERAL & \% EN VOLUMEN \\
\hline Cuarzo & 23.9 \\
\hline Plagioclasa (andesina) & 48.4 \\
\hline Hornblenda & 9.3 \\
\hline Biotita & 9.3 \\
\hline Feldespato potásico & 6.7 \\
\hline Clorita & 1.6 \\
\hline Accesorios & 0.8 \\
\hline
\end{tabular}

Parte del área donde aflora el Batolito Antioqueño está cubierta por un manto de suelos derivados de ceniza volcánica de $1.3 \mathrm{~m}$ de espesor medio, llegando en algunos lugares a $3 \mathrm{~m}$. La porosidad de este material, su elevada permeabilidad, su posición en superficie y su espesor hacen de él un elemento clave en el proceso de infiltración (Patiño et $a l ., 2000)$, y por ende genera un gran control en el proceso de erosión de los materiales que recubre. Ensayos preliminares realizados por medio de la filtración de agua destilada a través del horizonte Ade los andosoles derivados de estas capas de ceniza indican un descenso significativo del $\mathrm{pH}$.

\section{Resultados de Análisis de Aguas Superficiales}

La composición química del agua de los ríos está dominada por los productos solubles de la meteorización de los silicatos presentes en las rocas (Reid et al., 1981), de ahí que la tasa de meteorización química de las cuencas se puede inferir a partir de las características químicas del agua superficial. Los análisis químicos publicados por Geale \& Hermelin (1988) se hicieron sobre 33 muestras de aguas superficiales recogidas en cada una de las cuatro cuencas durante un periodo de un año con intervalos de 15 días, en sitios de buena mezcla y a una profundidad tal que no ocurriera resuspensión de sólidos del lecho del río (Geale \& Hermelin, 1988). Los resultados promedio se muestran en la tabla 3 .

Tabla 3. Composición Química Promedio (mg/l) de aguas superficiales, (Geale \& Hermelín, 1988)

\begin{tabular}{|l|c|c|c|c|}
\hline SOLUTO & Río Calderas & Río Tafetanes & Q. Jaguas & Q. Juanes \\
\hline $\mathbf{H}_{4} \mathbf{S i O} \mathbf{4}^{+3}$ & 12.6 & 12.38 & 23.13 & 29.96 \\
\hline $\mathbf{A l}^{+3}$ & 0.25 & 0.23 & 0.28 & 0.33 \\
\hline $\mathbf{F e}^{+\mathbf{3}}$ & 0.27 & 0.4 & 0.41 & 0.32 \\
\hline $\mathbf{C a}^{+\mathbf{2}}$ & 2.08 & 2.65 & 6.16 & 7.23 \\
\hline $\mathbf{M g}^{+\mathbf{2}}$ & 0.48 & 0.68 & 1.29 & 1.52 \\
\hline $\mathbf{N a}^{+}$ & 1.62 & 1.72 & 4.11 & 5.47 \\
\hline $\mathbf{K}^{+}$ & 0.82 & 0.96 & 1.29 & 1.71 \\
\hline $\mathbf{M n}^{+\mathbf{2}}$ & 0.015 & 0.031 & 0.044 & 0.032 \\
\hline $\mathbf{C l}^{-}$ & 2.94 & 2.56 & 2.65 & 2.63 \\
\hline $\mathbf{H C O}_{\mathbf{3}}^{-}$ & 15.74 & 20.88 & 40.77 & 52.09 \\
\hline
\end{tabular}


Las concentraciones de sílice, sodio y calcio presentan un comportamiento relativamente estable a lo largo del año, pero difieren entre cuenca y cuenca, aunque se aprecia un comportamiento similar entre las cuencas de los Ríos Tafetanes y Calderas y entre las de las Quebradas Juanes y Jaguas.

Esta agrupación coincide con la situación térmica de los dos grupos de cuencas, ya que en las zonas menos elevadas y de mayores temperaturas, como las Quebradas Juanes y Jaguas, las concentraciones llegan a triplicar a las de los Ríos Tafetanes y Calderas, ubicados a mayor altitud y con menores temperaturas.

Además del análisis químico de las aguas superficiales, se analizaron las aguas lluvia recolectadas por un periodo de seis meses en una estación pluviométrica cercana a las cuatro cuencas (tabla 4).

Tabla 4. Concentración Promedio de Solutos en las Aguas Lluvia para la Estación Pluviométrica de San Carlos (Geale \& Hermelin, 1988).

\begin{tabular}{|l|c|}
\hline SOLUTO & $\begin{array}{c}\text { Concentración } \\
(\mathbf{m g} / \mathbf{l})\end{array}$ \\
\hline $\mathbf{F e}^{+3}$ & 0.14 \\
\hline $\mathbf{C a}^{+2}$ & 0.48 \\
\hline $\mathbf{M g}^{+2}$ & 0.37 \\
\hline $\mathbf{N a}^{+}$ & 0.18 \\
\hline $\mathbf{K}^{+}$ & 0.47 \\
\hline $\mathbf{C I}^{-}$ & 2.79 \\
\hline $\mathrm{HCO}_{3}^{-}$ & 7.38 \\
\hline
\end{tabular}

\section{Perfil de Meteorización del Batolito Antioqueño}

El perfil de meteorización del Batolito Antioqueño es causado en mayor medida por la hidrólisis y oxidación de sus minerales primarios (Geale \& Hermelin, 1988), especialmente de los silicatos y específicamente de las plagioclasas, las cuales al transformarse generan como producto principal la caolinita debido a que su tasa de reacción con el agua es relativamente rápida (Colman, 1981, tomado de Pavich, 1986); se producen además ácido silícico, ácido carbónico y cationes disueltos ( $\mathrm{Ca}$ y $\mathrm{Na}$ principalmente), los cuales al ser lavados por el agua lluvia quedan en solución y son transportados hacia los ríos; esto explica que la concentración de calcio, sodio, magnesio y bicarbonato sea más alta en las aguas recolectadas de los ríos que en el agua lluvia.

En los suelos residuales de rocas cristalinas el nivel freático está controlado por la profundidad de meteorización que a su vez está relacionada directamente con su posición topográfica (Hoyos et al., 1985).

La sílice disuelta se origina por el rompimiento químico de la estructura de los silicatos presentes en la roca, causado por el reemplazamiento de cationes por los iones de hidrógeno provenientes principalmente de la disociación del ácido carbónico. Los cationes reemplazados migran, permitiendo que proceda la reacción y que los iones $\mathrm{H}^{+}$viajen desde la superficie hacia el interior de la estructura cristalina, sustituyendo a otros cationes (Geale \& Hermelin, 1988). Por su parte los cationes de calcio y sodio
Proceden principalmente de la meteorización química de las plagioclasas.

La meteorización química del Batolito Antioqueño ha generado un perfil compuesto principalmente por tres horizontes o zonas (Hoyos et al., 1985), de arriba abajo: a) zona somera, de uno a dos metros de espesor de color anaranjado a rojizo, compuesta por arcilla y limos arcillosos muy plásticos, parcialmente orgánicos, sin estructuras originales de la roca, equivalente a un suelo residual maduro de limos de baja permeabilidad (Patiño et al., 2000); ha sufrido bioturbación, proceso que ha destruido su estructura original; b) zona saprolítica de espesor variable, compuesta de limos arenosos y arenas limosas oxidadas, donde la textura original de la roca se conserva así como las estructuras originales de la roca: diques y venas de cuarzo, diaclasas, zonas de cizalladura heredadas; algunas veces presenta núcleos residuales de roca fresca; y c) zona de gruss, compuesta por arena y agregados poliminerálicos fácilmente disgregables, con la estructura original y textura de la roca fresca bien conservadas y núcleos de roca fresca mas grandes y mas abundantes que en la zona saprolítica; esta es la zona donde se encuentra al nivel freático. La roca fresca se encuentra inmediatamente debajo de la zona de gruss.

El espesor del saprolito del Batolito Antioqueño ha sido estudiado por Suescún (1966) en La Unión, donde encontró un espesor de $45 \mathrm{~m}$ bajo unos depósitos de arcilla sin llegar a la roca fresca. Feininger (1971) realizó estudios en Santa Rita cerca de Guatapé y en Puerto Velo (a unos 20 $\mathrm{km}$ al norte de la zona de San Carlos) donde 
encontró espesores promedio del saprolito de $31 \mathrm{~m}$ y $40 \mathrm{~m}$ respectivamente. Feininger \& Botero (1982) sugieren un espesor promedio de $34.2 \mathrm{~m}$ y un máximo de $80 \mathrm{~m}$. Page (1986) menciona espesores de saprolito del orden de $50 \mathrm{~m}$ en el área de Rionegro. Hoyos et al. (1985) muestran profundidades del saprolito muy variables las cuales tienden a aumentar significativamente de los valles a las cuchillas donde llegan a tener valores de $87 \mathrm{~m}$. Los mayores espesores fueron calculados a partir de métodos geoeléctricos en los altiplanos de Rionegro y de la Unión, donde el basamento puede estar en un intervalo de profundidad entre 40 y $200 \mathrm{~m}$. (Hoyos et al., 2000). Esta diferencia de valores se debe, entre otros, a que la profundidad y el tipo de perfil que resulta de la meteorización presentan grandes variaciones aun en un mismo tipo de roca, dependiendo principalmente de su litología y estructura, topografía y clima (Hoyos et al., 1985).

Según Page \& James (1981) la profundidad del saprolito es una combinación de las bajas tasas de erosión con las tasas normales de meteorización química. Aunque no se tienen valores concretos de la tasa del erosión del batolito, se deduce por el gran espesor del saprolito que es una tasa mucho menor que la tasa de meteorización.

El perfil de meteorización del Batolito Antioqueño está, en buena parte de la zona de estudio, recubierto por suelos derivados de cenizas volcánicas holocénicas (?) lo que hace que presenten propiedades ándicas (CORNARE, 1996) y por lo tanto puedan ser clasificados como Andisoles por el sistema Taxonómico Americano (USDA, 1999). El proceso dominante en este tipo de suelos es el de la meteorización y la transformación de minerales. El perfil modal de la zona de estudio (Consociación San Vicente) equivale a un Andisol típico (Hapupland típico, familia medial), que se caracteriza por presentar un horizonte Ap (00-30 $\mathrm{cm})$ de color negro, textura franco arenosa y estructura de bloques subangulares medios débilmente desarrollados; lo infrayace un horizonte $\mathrm{Bw}(70-105 \mathrm{~cm})$ ligeramente plástico con dos secciones de color pardo amarillento y amarillo rojizo, textura franco arcillo arenosa y franco arenosa y estructura de bloques subangulares medios, débilmente desarrollados; el horizonte mas profundo es un C $(105-150 \mathrm{~cm})$ de color amarillo rojizo y de textura franco arcillo arenosa (CORNARE, 1996).

Entre las cenizas volcánicas y el perfil de meteorización del Batolito Antioqueño se intercala a menudo una stone line (Hermelin, 1990), capa delgada de grava compuesta por cantos de cuarzo angular o redondeado según proceda de diques o de material aluvial, fragmentos de hidróxido de hierro, nódulos de gibbsita y fragmentos líticos, que indica un corto emplazamiento por flujo acuoso laminar en un clima diferente a las condiciones húmedas actuales. Sin embargo, la stone line (Fig. 2) muy pocas veces afecta al saprolito ya que generalmente se encuentra entre el material bioturbado y el perfil de cenizas.

Aunque en la zona no haya evidencias al respecto, en algunas partes del trópico húmedo se considera que la stone line, por estar ubicada en la parte superior del saprolito, lo protege de los procesos erosivos (Kadomura \& Hori, 1990 en Thomas 1994a)

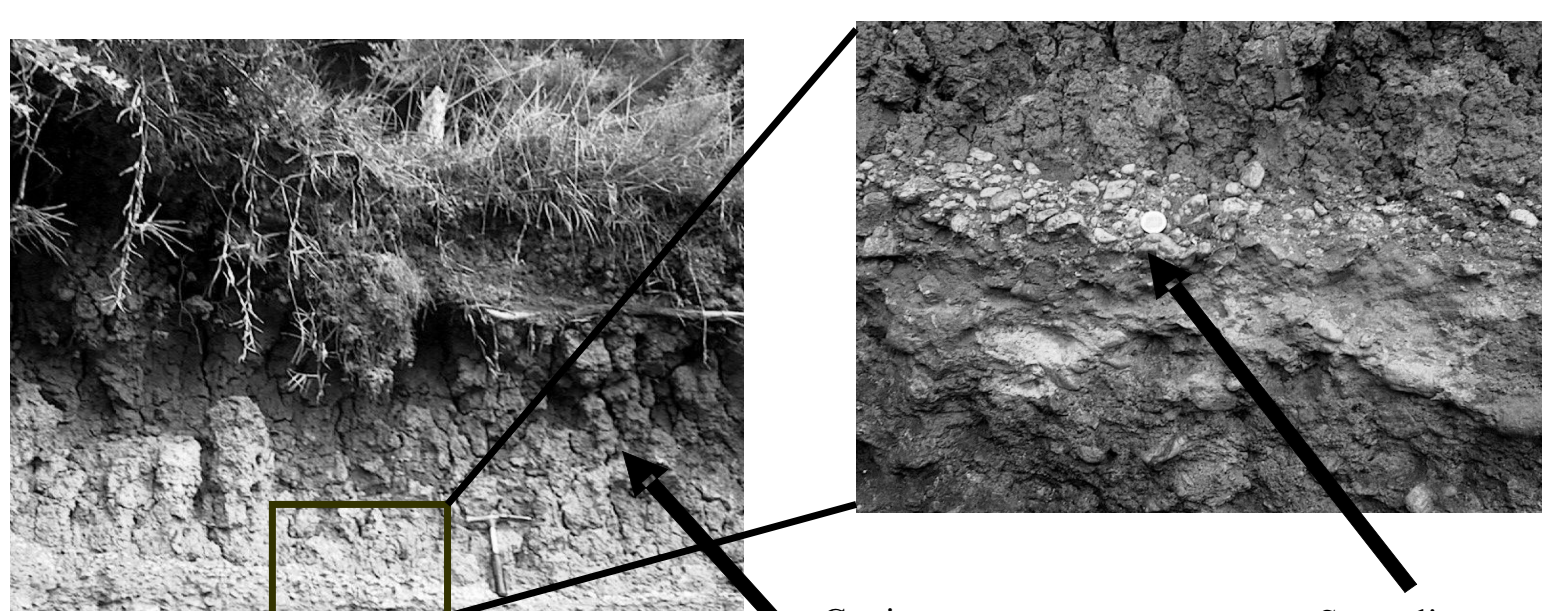

Cenizas

Volcánicas
Stone line

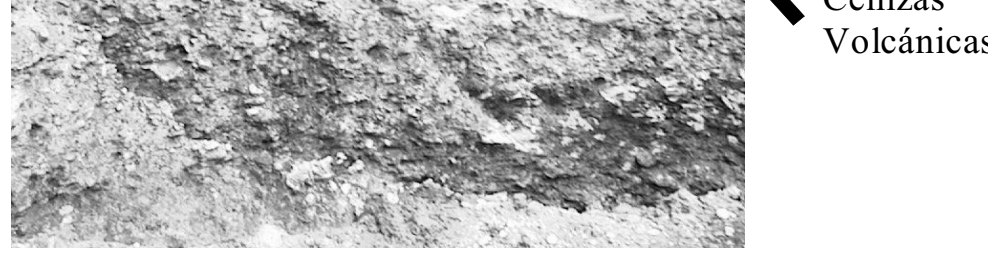

Figura 2. Stone line y cenizas volcánicas. Municipio de Rionegro, Oriente antioqueño. 
No obstante, la stone line es en sí misma evidencia de un corto periodo erosivo del Pleistoceno Superior. Otros eventos erosivos mucho mas importantes están evidenciados por la presencia de inselbergs o "peñoles" en áreas cercanas a la zona de estudio. Su formación, anterior al desarrollo de la superficie de erosión estudiada, se debe probablemente a zonas con diaclasas muy distanciadas donde las aguas meteóricas no pudieron penetrar y meteorizar la roca (Botero, 1963). Otra evidencia de erosión es la presencia de terrazas escalonadas que indican un descenso del nivel de las llanuras aluvial de corrientes como el Río Negro y sus afluentes. Estos dos últimos fenómenos, cuya edad se estudia en estos momentos, son mucho mas antiguos que el emplazamiento de la stone line.

\section{Tasas de Meteorización}

Se han realizado varios estudios sobre la determinación de la edad de saprolitos en diferentes climas y regiones del mundo como los de Pavich (1985,1989), Velbel (1985,1986), Dethier (1986), Cleaves (1970), Nahon (1970), Ollier (1996), Hanntjens \& Bleeker (1970) y Meybeck (1987), entre otros. El primero utiliza una metodología basada en el cálculo de la pérdida de solutos de la roca; éstos son transportados por las aguas subterráneas y de escorrentía. Los otros trabajos basan sus cálculos en balances de masa de las ecuaciones de transformación de la totalidad de los minerales primarios de las rocas en minerales secundarios (Thomas, 1994a), tomando en cuenta la proporción de cada mineral dentro de la roca. En la tabla 5 se dan lo valores encontrados por varios autores para las tasas de formación de saprolito por meteorización isovolumétrica, los cuales varían entre 2 a 50 metros por millón de años.

Tabla 5. tasa de formación de saprolito en diferentes zonas. Adaptado de Thomas (1994a) y Nahon (1991).

\begin{tabular}{|c|c|c|c|c|}
\hline ZONA DE ESTUDIO & AUTOR & METODO & $\begin{array}{l}\text { Precipitación } \\
\text { Promedio(mm) }\end{array}$ & $\begin{array}{l}\text { TASA } \\
\text { m/Ma }\end{array}$ \\
\hline Smoky Mountains & Velbel, 1985 & Balance de masas & - & 38 \\
\hline Southern Blue Ridge & Velbel, 1986 & Balance de masas & - & 37 \\
\hline $\begin{array}{l}\text { Varios tipos de roca, NW del } \\
\text { Pacífico, EEUU }\end{array}$ & Dethier, 1986 & Balance de masas & $750-4000$ & 33 \\
\hline $\begin{array}{l}\text { Monte Masanutten, Virginia, } \\
\text { EEUU }\end{array}$ & $\begin{array}{l}\text { Afifi y Bricker, } \\
1983\end{array}$ & Balance de masas & - & $2-10$ \\
\hline Granito, Piedemonte Sur, EEUU & $\begin{array}{l}\text { Pavich, } 1986 \\
\text { Pavich, } 1989\end{array}$ & $\begin{array}{l}\text { Pérdida en solución } \\
\text { Tiempo de residencia }\end{array}$ & 1040 & $\begin{array}{c}4 \\
20\end{array}$ \\
\hline Esquisto, Piedemonte de Baltimore & $\begin{array}{l}\text { Cleaves et al. , } \\
1970 \\
\text { Cleaves, } 1989\end{array}$ & $\begin{array}{l}\text { Balance de masas } \\
\text { Modelo de equilibrio }\end{array}$ & 735 & $\begin{array}{c}5-8 \\
25-48\end{array}$ \\
\hline $\begin{array}{l}\text { Granito, Noruega } \\
\text { Granito, E de Francia } \\
\text { Granito, S de Francia } \\
\text { Migmatita, S de Francia } \\
\text { Migmatita, N Costa de Marfil } \\
\text { Anfibolita, S de Francia } \\
\text { Basalto, Madagascar }\end{array}$ & Tardy, 1969 & $\begin{array}{l}\text { Pérdida de } \mathrm{Si} \\
\text { en solución }\end{array}$ & $\begin{array}{r}1250 \\
\\
850 \\
680 \\
680 \\
540 \\
640 \\
1500\end{array}$ & $\begin{array}{c}11.8 \\
19.2 \\
24.4 \\
10 \\
15.4 \\
14.7 \\
25\end{array}$ \\
\hline Granito, Costa de Marfil & $\begin{array}{l}\text { Leneuf, } 1959 \\
\end{array}$ & Pérdida en solución & - & $5-50$ \\
\hline Granito, Costa de Marfil & Boulangé, 1984 & - & - & 14 \\
\hline Chad & Gac, 1979 & - & - & 9.4 \\
\hline $\begin{array}{l}\text { Rocas Ultramáficas, Nueva } \\
\text { Caledonia }\end{array}$ & Trescases, 1975 & - & - & $29-47$ \\
\hline
\end{tabular}

La tasa de meteorización de rocas cristalinas estimada por Thomas (1994b) para zonas no tropicales (con latitudes de $36-39^{\circ} \mathrm{N}$ ) varía entre 20-50 m/Ma. Y aunque Nahon (1991) estima que estas tasas en climas tropicales húmedos están entre 14.3-50 m/Ma, valores muy similares a los de climas no tropicales, Thomas (1994b) plantea que para climas tropicales de regiones boscosas húmedas con temperaturas y niveles de precipitación dos a tres veces mayores que aquellos de zonas no tropicales, el flujo base y el aumento de temperatura podrían acelerar la tasa de formación de saprolito a valores de $60-150 \mathrm{~m} / \mathrm{Ma}$.

Dentro del cálculo del tiempo que se demora un determinado volumen de roca para convertirse en saprolito hay que tener en cuenta una gran cantidad de variables involucradas en el control del proceso de meteorización. Para proponer un método determinado se parte de una serie de suposiciones, que se plantean a continuación.

Toda el agua superficial recolectada 
estudio tiene su origen en la precipitación caída recientemente sobre la cuenca y no proviene de agua de fuentes profundas ni es agua que ha viajado desde otras cuencas (Cleaves, 1970); por esto se desprecia la escorrentía ya que casi toda la precipitación penetra en el suelo antes de llegar al río.

Las aguas lluvia responsables del proceso de hidrólisis sufren cambios de $\mathrm{pH}$ significativos al penetrar las diferentes zonas del saprolito.

En paisajes saprolíticos como el del Batolito Antioqueño, la meteorización química es isovolumétrica, es decir, se mantiene el mismo volumen del material parental aun después de la meteorización; por lo tanto no se espera cambio alguno ni en el volumen del paisaje ni en las geoformas (Cleaves \& Costa, 1979; Costa \& Cleaves, 1984 tomado de Velbel, 1986)

El clima ha sufrido cambios fuertes de temperatura y pluviosidad. En la zona de estudio se han presentado al menos dos épocas de erosión intensa: la primera (mucho mas antigua que la segunda) está evidenciada por la presencia de inselbergs o "peñoles" y la segunda está marcada por la presencia de la stone line, índice de un periodo relativamente corto de erosión superficial que no afectó drásticamente el perfil de meteorización estudiado.

La sílice disuelta presente en las aguas superficiales se debe únicamente al intercambio catiónico en la estructura de los minerales silicatados y no al cuarzo, pues aunque éste no es totalmente estable, en este caso se considera que no ha sido afectado por el proceso de meteorización.

A pesar de que White (1995) plantee que la tasa de meteorización disminuye con el tiempo debido en parte a la remoción de minerales reactivos, se considera en este estudio que la tasa de disolución de las especies es constante, debido en parte al fenómeno de levantamiento de la Cordillera Central, lo que permite que el contacto entre los minerales y la solución sea lo suficientemente largo para permitir una reacción continua (Pavich,1989).

El volumen de solución que atraviesa la parte meteorizable o reactiva del saprolito es constante. De esta manera la tasa de pérdida de solutos en solución también debe ser constante.

\section{Cálculo de la Tasa de Meteorización}

El cálculo de la tasa de formación del saprolito involucra el área y el caudal de cada cuenca y la cantidad de sólidos disueltos en las aguas superficiales (Tabla 3) menos los sólidos disueltos en el agua de lluvia (Tabla 4). Así la tasa de meteorización, es decir la masa removida en cada cuenca por disolución mineral, se obtiene con la ecuación 1 (adaptada de Geale \& Hermelin, 1988):

$$
\begin{aligned}
& \mathrm{T}_{\mathrm{M}}=\mathrm{Ci} * \mathrm{Q} * 31536000 \text { (seg-año) } \\
& \text { ecuación } 1 \\
& \mathrm{~T}_{\mathrm{M}-\mathrm{kg}}=\left(\mathrm{Ci}^{*} 0.001\right) * \mathrm{Q} * 31536000(\mathrm{seg}- \\
& \text { año)/A ecuación la }
\end{aligned}
$$

Donde:

$\mathrm{T}_{\mathrm{M}}$ : tasa de meteorización química anual en $\mathrm{g} / \mathrm{cm}^{2}$-año (remoción de solutos)

$\mathrm{C}_{\mathrm{i}}$ : Concentración promedio anual de la especie $\mathrm{i}$ en $\mathrm{g} / \mathrm{l}$

$\mathrm{Q}$ : Caudal procedente de la cuenca, $1 / \mathrm{s}$

A: Área de la cuenca, $\mathrm{cm}^{2}$

$\mathrm{T}_{\mathrm{M}-\mathrm{kg}}$ : tasa de meteorización química anual en kg/ha-año (remoción de solutos)

Si se conoce la remoción o pérdida de solutos [D] en $\left(\mathrm{g} / \mathrm{cm}^{3}\right)^{\mathrm{q}}$ ue se genera en el proceso de meteorización para pasar de roca fresca a saprolito y el área de la cuenca, con la ecuación 2 (Pavich, 1981) se puede determinar el material removido [MR] en gramos necesario para transformar un $\mathrm{cm}^{3}$ de roca en saprolito:

$$
\mathrm{D}\left(\mathrm{g} / \mathrm{cm}^{3}\right) * \underset{\text { ecuación } 2}{\mathrm{~V}\left(\mathrm{~cm}^{3}\right)}=M R(\mathrm{~g})
$$

Donde:

D: Remoción o pérdida de solutos en $\mathrm{g} / \mathrm{cm}^{3}$ $1 \mathrm{~cm}$ de espesor

$\mathrm{V}$ : volumen en $\mathrm{cm}^{3}$ : área de la cuenca por

MR: material removido en $\mathrm{cm} /$ año

Conociendo el material removido [MR], el tiempo requerido para remover $1 \mathrm{~cm}$ esta masa en solución viene dado por la ecuación 3 :

$$
\begin{aligned}
& M R \quad \begin{array}{l}
(g) / T_{\mathrm{M}}=\text { a ̃̃ o s } / \mathrm{cm} \\
\text { ecuación } 3
\end{array} \\
& \text { Para determinar la tasa de }
\end{aligned}
$$
transformación de roca a saprolito en metros por millón de años $(\mathrm{m} / \mathrm{Ma}$ ) se aplica la ecuación 4:

$10000 /($ años $/ \mathrm{cm})=T_{\mathrm{Ma}}(\mathrm{m} / \mathrm{Ma})$

Donde:

$$
\text { ecuación } 4
$$

$\mathrm{T}_{\mathrm{Ma}}$ : tasa de meteorización química en $\mathrm{m}$ / $10^{6}$ años $[\mathrm{m} / \mathrm{Ma}]$ (remoción de solutos)

Finalmente se determina el tiempo necesario para formar los $\mathrm{H}$ m de espesor con la ecuación 5:

$$
\mathrm{H}(\mathrm{m}) / \mathrm{T}_{\text {м a a }}(\mathrm{m} / \mathrm{M} \text { a })
$$

Donde:

$H$ : espesor del saprolito en metros. Para el estudio se van a utilizar dos valores para esta variable:

Un máximo espesor promedio de saprolito de 80 m (Feininger \& Botero (1982); Hoyos et al., (1985); Hoyos et al., (2000))

Un máximo espesor de saprolito de $200 \mathrm{~m}$ (Hoyos etal., 2000)

\section{CASO 1}

La cantidad de sólidos disueltos en las aguas superficiales menos el aporte de sólidos presentes en las aguas de lluvia puede ser utilizada para calcular la tasa a la cual un determinado volumen de roca es alterado al mismo volumen de saprolito (Pavich, 1986). Se supone el mismo 
volumen de roca basado en la teoría de "meteorización isovolumétrica" de Millot, pero teniendo en cuenta que sí hay una pérdida de solutos evidenciada en el cambio de densidad de la roca fresca $\left(2.6 \mathrm{~g} / \mathrm{cm}^{3}\right)$ a la densidad del saprolito $(1.5$ $\mathrm{g} / \mathrm{cm}^{3}$ )' lo cual implica que a raíz de la meteorización hay una remoción o pérdida de masa de $1.1 \mathrm{~g} / \mathrm{cm} 3$ (equivalente al término [D] de la ecuación 2).
De esta manera, $\mathrm{C}_{\mathrm{i}}$ de la ecuación 1 es la suma de todos los componentes encontrados en las aguas superficiales de cada cuenca (Tabla 3) menos la suma de los componentes encontrados en las aguas de lluvia (Tabla 4).

Sustituyendo los valores en las ecuaciones respectivas se obtienen los resultados de la Tabla 6.

Tabla 6. Resultados utilizando el total de sólidos disueltos en las aguas superficiales.

\begin{tabular}{|c|c|c|c|c|}
\hline & Río Calderas & Río Tafetanes & Q. Jaguas & Q. Juanes \\
\hline $\mathbf{T}_{\mathbf{M}-\mathrm{kg}}(\mathrm{kg} / \mathrm{ha}$-ä̈o) & 752.66 & 916.95 & 1770.25 & 1058.21 \\
\hline \multicolumn{1}{|c|}{$\mathbf{T}_{\mathbf{M a}}(\mathrm{m} / \mathrm{Ma})$} & 68.4 & 83.4 & 161 & 96.2 \\
\hline $\begin{array}{l}\text { Ä̈os (para formar } \\
80 \text { m de saprolito) }\end{array}$ & 1169184 & 959703 & 497106 & 831590 \\
\hline $\begin{array}{l}\text { Ä̈os (para formar } \\
200 \text { m de saprolito) }\end{array}$ & 2922961 & 2399257 & 1242765 & 2078974 \\
\hline
\end{tabular}

\section{$\mathrm{CASO} 2$}

Teniendo en cuenta que el mineral índice de la meteorización para este tipo de roca es la plagioclasa y sabiendo que sus productos principales son $\mathrm{Na}$ y $\mathrm{Ca}$ además de $\mathrm{Si}$, se utilizaron nuevamente las ecuaciones 1,2 y 3 pero sustituyendo el valor de $C_{\mathrm{i}}$ por la suma de $\mathrm{Na}$, Ca y $\mathrm{Si}$ solamente. Con esto se obtuvieron los resultados de la Tabla 7 .

Tabla 7. Resultados utilizando la suma de $\mathrm{Ca}$, Na y Si disueltos en las aguas superficiales.

\begin{tabular}{|l|c|c|c|c|}
\hline & Río Calderas & Río Tafetanes & Q. Jaguas & Q. Juanes \\
\hline \multicolumn{1}{|c|}{$\mathbf{T}_{\mathbf{M - k g}}$ (kg/ha-aÿo) } & 470.77 & 480.88 & 848.28 & 496.69 \\
\hline \multicolumn{1}{|c|}{$\mathbf{T}_{\mathbf{M a}}$ (m/Ma) } & 42.8 & 43.7 & 77.1 & 45.2 \\
\hline $\begin{array}{l}\text { Aÿos (para formar } \\
80 \text { m de saprolito) }\end{array}$ & 1869274 & 1829996 & 1037394 & 1771722 \\
\hline $\begin{array}{l}\text { Ä̈os (para formar } \\
200 \text { m de saprolito) }\end{array}$ & 4673186 & 4574990 & 2593485 & 4429304 \\
\hline
\end{tabular}

\section{CASO 3}

Ya que puede haber una variación en la cantidad de $\mathrm{Ca}$ y $\mathrm{Na}$ aportada por la meteorización debido a una posible contaminación externa generada por factores como abonos o nutrientes de la vegetación, se toma en cuenta únicamente la sílice para el cálculo de la tasa de meteorización. Esta decisión esta sustentada en varias afirmaciones de diversos autores entre las que se encuentran:

la sílice es uno de los productos principales 
de la meteorización química de las rocas graníticas, que se origina únicamente por la meteorización de silicatos (Stallard \& Edmond, 1983);

La sílice está en equilibrio dinámico con la biomasa (Lovering, 1959; Miller, 1963 tomado de Cleaves, 1970), es decir, que la absorción de sílice por parte de los organismos es insignificante los organismos es insignificante

El aporte de sílice vía precipitación es despreciable (Stallard \& Edmond, 1983).

Por lo anterior se podría considerar a la sílice como el indicador más confiable de los cationes resultantes de la meteorización que se hallan disueltos en las aguas superficiales. De esta las ecuaciones sustituyendo $\mathrm{C}_{\mathrm{i}}$ únicamente por el

Tabla 8. Resultados utilizando sólo el Si disuelto en las aguas superficiales.

\begin{tabular}{|c|c|c|c|c|}
\hline & Río Calderas & Río Tafetanes & Q. Jaguas & Q. Juanes \\
\hline $\mathbf{T}_{\mathbf{M}-\mathrm{kg}}(\mathrm{kg} / \mathrm{ha}-\mathrm{aÿo})$ & 379.27 & 370 & 599.29 & 354.31 \\
\hline $\mathbf{T}_{\mathbf{M a}}(\mathrm{m} / \mathrm{Ma})$ & 34.5 & 33.6 & 54.5 & 32.2 \\
\hline $\begin{array}{c}\text { Ä̈os (para formar } \\
80 \text { m de saprolito) }\end{array}$ & 2320274 & 2378404 & 1468408 & 2483722 \\
\hline $\begin{array}{c}\text { Ä̈os (para formar } \\
200 \mathrm{~m} \text { de saprolito) }\end{array}$ & 5800685 & 5946009 & 3671020 & 6209305 \\
\hline
\end{tabular}

manera se utilizaron nuevamente las ecuaciones sustituyendo $\mathrm{C}_{\mathrm{i}}$ únicamente por el valor del Si de la Tabla 3, obteniendo con esto los resultados de la Tabla 8

\section{Análisis de Resultados}

Un factor que puede alterar los resultados es el hecho de que el análisis químico del agua lluvia se llevó a cabo sólo durante seis meses. Posiblemente si se tuviesen análisis de todo un año o mas se obtendrían resultados más confiables; además, esto permitiría evaluar la incidencia de los cambios meteorológicos anuales, especialmente en cuanto a la precipitación.

A pesar de que Velbel (1986) afirme que no es necesario incluir la biomasa en los cálculos por estar en un estado estable y constante y por lo tanto desprecie el aporte y la retención de solutos como nutrientes por parte de la vegetación, se recomienda tener en cuenta que un porcentaje, aunque bajo, de los solutos disueltos en el agua superficial puede provenir de aportes hechos por la descomposición de la capa vegetal, así como de aportes artificiales provenientes de abonos. Esto puede significar que los resultados obtenidos de sólidos disueltos sean algo más elevados que el resultado real.

La cobertura vegetal y la temperatura (asociadas a la altura sobre el nivel del mar) también son factores incidentes en el grado de meteorización química: por ejemplo la cuenca de la Quebrada Jaguas, que presenta poca altura respecto a las otras cuencas, evidenció la mayor tasa de meteorización. Hay que tener en cuenta además que el porcentaje de cobertura de vegetación ha variado a través de los años, especialmente en los últimos 50 años, debido al cambio en el tipo e intensidad de las prácticas pastoriles y agrícolas.

Las cuencas muy grandes (mayores de 20 ha), como las del Río Calderas, Río Tafetanes y Quebrada Jaguas, pueden generar resultados poco confiables (Jurado, 1988). Por lo anterior se escogieron los datos de la Quebrada Juanes como los valores mas representativos del estudio.

Se puede apreciar que la Quebrada Juanes, a pesar de tener la mayor cantidad de sílice disuelta presenta la menor tasa de meteorización. Esto se debe a que la relación de Area/Caudal es casi dos veces la de las otras cuencas, indicando con esto que presenta un área muy grande respecto al caudal; esto se traduce en una menor cantidad de solutos por unidad de área respecto a las otras tres cuencas.

Los suelos derivados de las capas de ceniza volcánica que fueron depositadas sobre el saprolito y las condiciones climáticas favorables permitieron el desarrollo de una vegetación boscosa que cumple una función protectora respecto a los procesos erosivos; la edad máxima de las cenizas volcánicas que cubren el Batolito Antioqueño fue estimada en $6.5 \mathrm{Ma}$ con el método de trazas de fisión en zircones de origen volcánico (Toro, 2004, comunicación oral), su depositación indica la edad mínima de una etapa en la que se favorecen las condiciones de meteorización profunda. La formación de los "peñoles" sería anterior a esa fecha.

Aunque los valores obtenidos para la 
edad de la meteorización del Batolito Antioqueño concuerden con los esperados según la edad de las cenizas volcánicas, se recomienda realizar nuevos estudios geoquímicos completos de otras cuencas pequeñas ubicadas sobre el batolito, esta vez aplicando el método del balance geoquímico de masas basado en las reacciones de transformación de los minerales primarios durante el proceso de meteorización. Lo anterior permitiría corroborar y mejorar los datos obtenidos en este estudio.

Además del método del balance geoquímico sería conveniente aplicar otros métodos para corroborar la edad de meteorización, como el paleomagnetismo (utilizado parcialmente por Page \& James, 1981, para determinar la edad relativa de las terrazas del Río Negro), el uso de isótopos cosmogénicos como Al y Be (Heismath et al., 1999) y el uso de isótopos estables como $\mathrm{O}$ y $\mathrm{H}$ (Bird et al., 1992) y Sr/Ca (Wright, 1988).

\section{Conclusiones}

Es difícil establecer cuál de los tres casos es el más adecuado para determinar la edad de la meteorización máxima promedio del Batolito Antioqueño, no sólo por la incidencia de todos los factores antes mencionados, sino por la diferencia de opiniones de los especialistas respecto a las cifras más representativas de la cantidad de solutos removidos en el proceso de formación del saprolito.

A pesar de lo anterior, se considera tentativamente que los datos obtenidos mas confiables son los del caso 3, en el cual sólo se tomó en cuenta la sílice para estimar la tasa de meteorización. Para condiciones similares a las de la cuenca de la Quebrada Juanes se requeriría un tiempo de 6.2 Ma para formar 200m de saprolito. Este dato es compatible con la edad máxima de las cenizas volcánicas mas antiguas que cubren y protegen el saprolito.

Debido a que el valor de los sólidos disueltos utilizado no incluye los sólidos disueltos aguas abajo de la toma de la muestras, se considera que los valores obtenidos para las tasas de meteorización obtenidas son probablemente menores que los valores reales.

Los altiplanos de la Cordillera Central están ubicados a alturas diferentes sobre el nivel del mar y según Page \& James (1981) fueron generados entre hace 15 y 1 millón de años. En cuanto a su formación hay dos hipótesis principales, la primera plantea que estas superficies se formaron por sucesivos levantamientos seguidos por periodos de quietud (Page \& James, 1981) durante los cuales se generaron aplanamientos por erosión fluvial. La segunda hipótesis plantea una sola superficie original separada en varios fragmentos situados ahora a diferentes alturas por juego vertical de fallas durante el levantamiento (Hermelin, comunicación oral, 2004). Los datos obtenidos para la edad del espesor máximo del saprolito se enmarcan dentro del orden de magnitud de las edades planteadas para estas superficies de erosión. Estudios mas detallados en curso sobre la diferencia en profundidad y de la edad de los perfiles de meteorización del batolito deberían permitir despejar las incógnitas que aún dejan las hipótesis respecto a la formación de las superficies de erosión en la zona estudiada.

\section{Citaciones Bibliografias}

Bird, M., Longstaff, F. Fyfe, W. \& Bildgen, P. 1992. Oxygen-isotope systematics in a multiphase weathering system in Haiti. Geochimica ef Cosmochimica Acta, 56: 2831-2838.

Botero, A. (1963). Contribución al Conocimiento de la Geología de la Zona Central de Antioquia. Anuales de la Facultad de Minas, No. 57. Medellín: 101p.

Cleaves, E., Godfrey, A. \& Bricker, O. 1970. Geochemical Balance of a Small Watershed and its Geomorphic Implications. In: Geological Society of America Bulletin, 81:3015-3032.

Colman, S. \& Dethier, D. 1986. An Overview of Rates of Chemical Weathering. In: COLMAN, S. e DETHIER, D. (org.) Rates of Chemical Weathering of Rocks and Minerals. Academic Press, Orlando: 1-17.

Cornare. 1996. Uso Potencial del Suelo en los Municipios del Altiplano del Oriente Antioqueño (rionegro, Marinilla, San Vicente, El Carmen del Viboral, Guarne, El Retiro, La Ceja, El Santuario y La Unión). Bogotá. $231 \mathrm{p}$.

Dethier, D. 1986. Weathering Rates and the Chemical Flux from Catchments in the Pacific Northwest, U.S.A. In: COLMAN, S. e DETHIER, D. (org.) Rates of Chemical Weathering of Rocks and Minerals. Academic Press, Orlando: 503-530.

Drever, J.I. \& Clow, D. W. 1995. Weathering Rates in Catchments. In: WHITE, A.F. e BRANTLEY, S. L. Chemical Weathering Rates of Silicate Minerals. Mineralogical Society of America. Reviews in Mineralogy, Washington, 31: 463-483.

Feininger, T. 1971. Chemical Weathering and Glacial Erosion of Crystalline Rocks and the Origin of Till. U.S. Geological Survey Prof. Paper 750-C: C65-C81.

Feininger, T. \& Botero, G. 1982. The Antioquian Batholith, Colombia. Publicaciones Geológicas Especiales del INGEOMINAS, (12), Bogotá-Colombia: 50p.

Geale, B. \& Hermelin, M. 1988. Aguas Subterráneas y Meteorización Química en varias Cuencas del Oriente Antioqueño. Memorias III Simposio Colombiano de Hidrogeología, Bogota: 129-150.

Haantjens, H. A. \& Bleeber, P. 1970. Tropical Weathering in the Territory of Papua and New Guinea. Australian Journal Soil Research, 8: 157-177.

Heimsath, A. H., Dietrich, W. E., Nishiizumi, K. \& Finkel, R. C. 1999 Cosmogenic nuclides, 
topography, and the spatial variation of soil depth. Geomorphology, 27 (1-2): 151-172.

Hermelin, M. 1993. Stone lines in the Antioquia Highlands. Quaternary of South America and the Antarctica Peninsula, 8: 137-156.

Hoyos, F.; Hermelin, M. \& Toro, G. 1985. Régimen de aguas subterráneas en suelos profundos derivados de rocas ígneas y metamórficas en la Cordillera Central de Colombia. In: II Simposio Colombiano de Hidrogeología. Bogotá: 107-125.

Hoyos, F.; Munera, J. C.; Arias, D. E. \& Vélez, M. V. 2000. Investigación de Aguas Subterráneas, Región Valle de San Nicolás. Convenio de Cooperación en Ciencia y Tecnología, CORNARE Universidad Nacional de Colombia, 44599. Medellín: 71p.

Jurado M., J. L. 1988. Componente Hidrológico y Meteorización Química en una Cuenca del Batolito Antioqueño. Trabajo de Grado, Postgrado de Recursos Hidráulicos Universidad Nacional de Colombia sede Medellín: 186p.

Lermam, A. 1988. Weathering Rates and Major Transport Processes an Introduction. In: LERMAN, A. E MEYBECK, M. (org.) Physical and Chemical Weathering in Geochemical Cycles. Kluwer Academic Publishers: 1-10.

Meybeck, M. 1987. Global Chemical Weathering of Surficial Rocks Estimated from River Dissolved Loads. American Journal of Science, 287: 401-428

Nahon, D. B. 1991. Introduction to the Petrology of Soils and Chemical Weathering. John Wiley and Sons: $313 \mathrm{p}$.

Page \& James. 1981. The Antiquity of the Erosion Surfaces and Late Cenozoic deposits near Medellin, Colombia: Implications to Tectonics and Erosion Rates. In: Revista CIAF, 6 (1-3): 421-454.

Page, W. 1986. Geología Sísmica y Sismicidad del Noroeste de Colombia. ISA, INTEGRAL, Woodward Clyde Consultants: $155 \mathrm{p}$.

Patiño, J. E. 2000. Toward a Chronology of the Northern Central Cordillera Plateaus, Colombia. Report, Course of Advanced Geomorphology. Universidad EAFIT. Medellín: 21p.

Pavich, M. 1986. Processes and Rates of Saprolite Production and Erosion on a Foliated Granitic Rock of the Virginia Piedmont. In: COLMAN, S. e Dethier, D. (org.) Rates of Chemical Weathering of Rocks and Minerals. Academic Press, Orlando: 551-590.

Reid, J. M.; Macleod, D. A. And Cresser, M. S. 1981. The Assessment of Chemical Weathering Rates within an Upland Catchments in NorthEast Scotland. In: Earth Surface Processes and Landforms, 6: p. 447-457.

Stallard, R. F. Y Edmond, J. M. 1983. Geochemistry of the Amazon 2. The Influence of Geology and Weathering Environment on the Dissolved Load. Journal of Geophysical Research, 88 (C14): 9671-9688

Stallard, R. F. Y Edmond, J. M. 1987. Geochemistry of the Amazon 3. Weathering Chemistry and Limits to Dissolved Inputs. Journal of Geophysical Research, 92 (C8): 8293-8302

Suescun, D. 1966. Estudio de las Arcillas Industriales en la Zona Central de Antioquia. Boletín Geológico, 14 (1-3): 5 53.

Thomas, M. F. 1994a. Ages and Geomorphic Relationships of Saprolite Mantles. In: Robinson, D. A. E Williams, R. B. G. (org.) Rock Weathering and Landform Evolution. John Wiley and Sons: 287-301.

Thomas, M. F. 1994b. Geomorphology in the Tropics: a Study of Weathering and Denudation in Low Latitudes. John Willey \& Sons. Chichester: 460p.

Usda. 1999. Soil Taxonomy: A basic system of soil classification for making and interpreting soil surveys. Agriculture Handbook, Number 436. Second Edition. 871 p.

Velbel, M. A. 1985. Geochemical Mass Balances and Weathering Rates in Forested Watersheds of the Southern Blue Ridge. American Journal of Science, 285: 901930.

Velbel, M. A. 1986. The Mathematical Basis for Determining Rates of Chemical and Geomorphic Processes in Small Forested Watersheds by Mass Balance: Examples and Implications. In: COLMAN, S. E DETHIER, D. (org.) Rates of Chemical Weathering of Rocks and Minerals. Academic Press, Orlando: 439-451.

Williams, A.G. \& Ternan, J. L. 1979. Hydrological pathways and granite weathering on Dartmoor. Plymouth: School of Environmental Sciences: $27 \mathrm{p}$.

White, F. Y Brantley, S. L. 1995. Chemical Weathering Rates of Silicate Minerals: an Overview. In: White, A.F. e Brantley, S. L. Chemical weathering rates of silicate minerals. Mineralogical Society of America. Reviews in Mineralogy, Washington, 31: 1-22.

Wright, R. 1988. Influence of Acid Rain on Weathering Rates. In: Lerman, A. E Meybeck, M. (org.) Physical and Chemical Weathering in 